\title{
Development of the Spin-valve transistor
}

\author{
D.J. Monsma, R. Vlutters, T. Shimatsu, E.G. Keim*', R.H. Mollema, and J.C. Lodder \\ Information Storage Technology Group, MESA Research Institute, ${ }^{*}$ Center of Materials Research, \\ University of Twente, 7500AE Enschede, The Netherlands
}

\begin{abstract}
As the easiest experimental approach, GMR (Giant Magnetoresistance) is usually measured using the Current in Plane (CIP)-GMR. The spin-valve transistor has previously been presented as a spectroscopic tool to measure Current Perpendicular to the Planes (CPP)-GMR. Hot electrons cross the magnetic multilayer base quasi-ballistically and the number reaching the collector depends exponentially on the perpendicular hot electron mean free path. Collector current changes of $390 \%$ at $77 \mathrm{~K}$ have already been measured. Apart from the substantial fundamental value, such properties may be useful for sensor applications. The electron energy range fills the gap between the Fermi surface transport in resistance measurements and other hot electron techniques such as spin polarised electron energy loss spectroscopy (SPEELS). The preparation problem of the spin-valve transistor and metal base transistor structures in general, the deposition of a device quality semiconductor on top of a metal, has now been tackled by bonding of two semiconductor substrates during vacuum deposition of a metal: an excellent bond is achieved at room temperature. TEM photos show a continuous buried metal film. Apart from preparation of various metal base transistor like structures, many other fields may benefit from this new technique.
\end{abstract}

\section{INTRODUCTION}

The discovery of giant magnetoresistance in magnetic multilayers [1], more appropriately called the spin-valve effect [2], has led to a large number of studies on metallic systems, motivated both by the enormous potential of the effect for magnetic sensors and the fundamental interest of the underlying principles. These efforts have resulted in magnetic multilayers that show considerable resistance change (of the order of $10 \%$ ) in fields useful for read head applications (20 Oe or $1.6 \mathrm{kA} / \mathrm{m}$ ) and magnetic RAMs. Usually, the resistance is measured with the current in the planes of the layers. From a fundamental point of view however, the CIP configuration suffers from several drawbacks: the CIP magnetoresistance (MR) is diminished by shunting and channeling and diffusive surface scattering reduces the MR for sandwiches and thin multilayers.

Moreover, fundamental parameters of the effect, such as the relative contributions of interface and bulk spin dependent scatterings are difficult to obtain using the CIP geometry. Measuring with the current perpendicular to the planes (CPP) solves most of these problems, mainly because the electrons cross all magnetic layers, but the perpendicular resistance of the ultra thin multilayers is too small to be measured by ordinary techniques. The first CPP-MR experiments were

Manuscript submitted April 24, 1997

D.J. Monsma 31-53-4893355 fax. -3343 D.J.Monsma@el.utwente.nl, This work was supported by the European research program Brite Euram N'7192, BRE2-0546. reported on $\mathrm{Co} / \mathrm{Ag}$ multilayers [3], where the multilayer was sandwiched between superconducting $\mathrm{Nb}$ leads. The use of microfabrication techniques for $\mathrm{CPP}$ measurements from $4.2 \mathrm{~K}$ to $300 \mathrm{~K}$ was firstly shown for $\mathrm{Fe} / \mathrm{Cr}$ multilayers [4], where the multilayers were etched into micro pillars to obtain a relatively large resistance (a few $\mathrm{m} \Omega$ ). Both types of measurements have confirmed the larger MR effect for the CPP configuration. Experiments using electrodeposited nanowires demonstrated CPP-MR at RT [5],[6] and recent experiments with etched grooved substrates introduced the so-called CAP (Current with an Angle to the Planes)configuration, for which also an increase of the spin-valve effect has been observed as compared to its CIP counterpart [7]-[9]. The spin-valve transistor has been presented as a new magnetic field sensor and measurement tool based on perpendicular hot electron transport in a spin-valve multilayer [10]. Here, a spin-valve multilayer serves as a base region of an n-silicon metal base transistor (MBT) structure, showing a very large change $(215 \%$ at $77 \mathrm{~K})$ in collector current in 500 Oe $(40 \mathrm{kA} / \mathrm{m})$, using a $(\mathrm{Co} / \mathrm{Cu})_{4}$ multilayer. The hot nature of the electrons and the possibility to vary the electron energy accurately in a range of about $0.2-3 \mathrm{eV}$, raises possibilities for fundamental research of the spin-valve effect and may lead to unforeseen effects and new spin-valves. In contrast to usual conduction electrons which are sensitive to the density of states (DOS) at the Fermi energy $\left(E_{F}\right)$, the hot electrons are sensitive to the DOS above $E_{F}$.

Manipulation of electron energy may offer important insights into the relative importance of the band structure (DOS) and scattering potentials, which are understood to form the basis of the scattering asymmetry between majority and minority conduction electrons. Currently electron energy spectroscopy in transport of magnetic systems receives increased interest as an additional tool to study the fundamentals of the spin-valve effect.

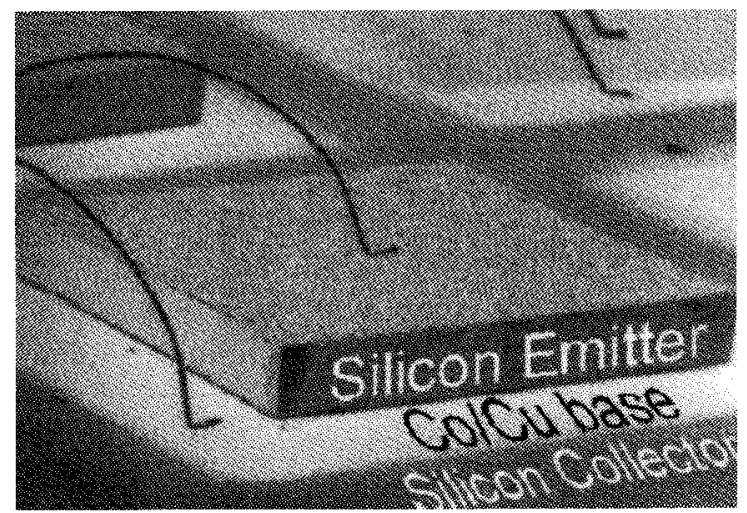

Fig. 1 SEM picture showing the collector, multilayers base region and direct-bonded (in air) emitter. Aluminium wires are connected using ultrasonic wire bonding. 
Techniques such as spin polarised tunneling, scanning electron microscopy with polarisation analysis (SEMPA), spin polarised low electron energy diffraction (SPLEED) and spin polarised electron energy loss spectroscopy (SPEELS) are under investigation in various laboratories [11]-[17].

\section{PRINCIPLE}

A SEM picture of one of the realised spin-valve transistor structures in shown in Fig. 1. A $(\mathrm{Cu} 2 \mathrm{~nm} / \mathrm{Co} 1.5 \mathrm{~nm})_{4}$ multilayer base is rf-sputtered onto the Si(100) collector substrate. The emitter has been direct-bonded in air to the base multilayer. The emitter is negatively biased (forward) using a DC current source, the collector substrate is in reverse (positive voltage bias), in common base. The base area is 2.7 $\times 2.7 \mathrm{~mm}^{2}$, the emitter area $1.6 \times 1.6 \mathrm{~mm}^{2}$.

\section{HOT ELECTRON TRANSPORT}

A schematic energy band diagram of the bonded $\mathrm{Co} / \mathrm{Cu}$ spin-valve transistor is shown in Fig. 2. The emitter (collector) barrier height is about $0.7 \mathrm{eV}(0.6 \mathrm{eV})$. The emitter bias accelerates the electrons over the emitter barrier, after which they constitute the hot, quasi-ballistic electrons in the base. The probability of passing the collector barrier is limited by collisions in the base, which affect their energy and trajectory, by optical phonon scattering in the semiconductors and by quantum mechanical reflections at the base-collector interface. For a metal base transistor with a single metal base film, this can be expressed by the common base current transfer ratio or current gain $\alpha_{0}=\left(\mathrm{J}_{c}-\mathrm{J}_{\text {leak }}\right) / \mathrm{J}_{\mathrm{e}}=\alpha_{e} \alpha_{c} \alpha_{\text {qm }} \mathrm{e}^{-\mathrm{W} / 2}$, in which $\alpha_{\mathrm{e}}, \alpha_{\mathrm{c}}$ and $\alpha_{\mathrm{qm}}$ represent emitter efficiency, collector efficiency and quantum mechanical transmission, respectively. $W$ is the base width and $\lambda$ is the hot electron mean free path (MFP) in the base. The factor $\mathrm{e}^{-\mathrm{W} / \lambda}$ represents the probability of transmission of the hot electrons through the base. $J_{c}$ is the total collector current, $J_{\text {leakage }}$ is the collector leakage current, determined by the reverse-biased collector Schottky barrier and $J_{c}$ is the injected emitter current. In the spin-valve transistor under consideration, the collector current of the $\mathrm{Co} / \mathrm{Cu}$ spin-valve transistor depends exponentially on the spin dependent hot electron mean free paths $\lambda_{\downarrow(\uparrow)}$ in the base. As a result from the perpendicular trajectory, the exponential mean free path (MFP) dependence and possibly also the electron energy, the collector current variation is large, as shown in Fig. 3.

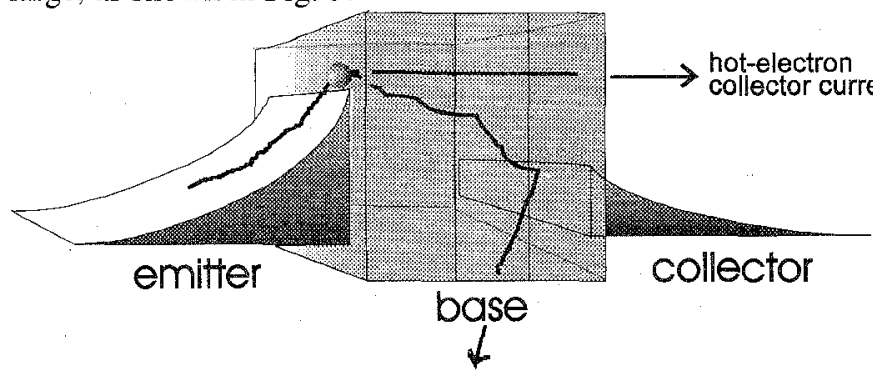

FIG. 2. Schematic energy band diagram of the spin-valve transistor under forward bias.

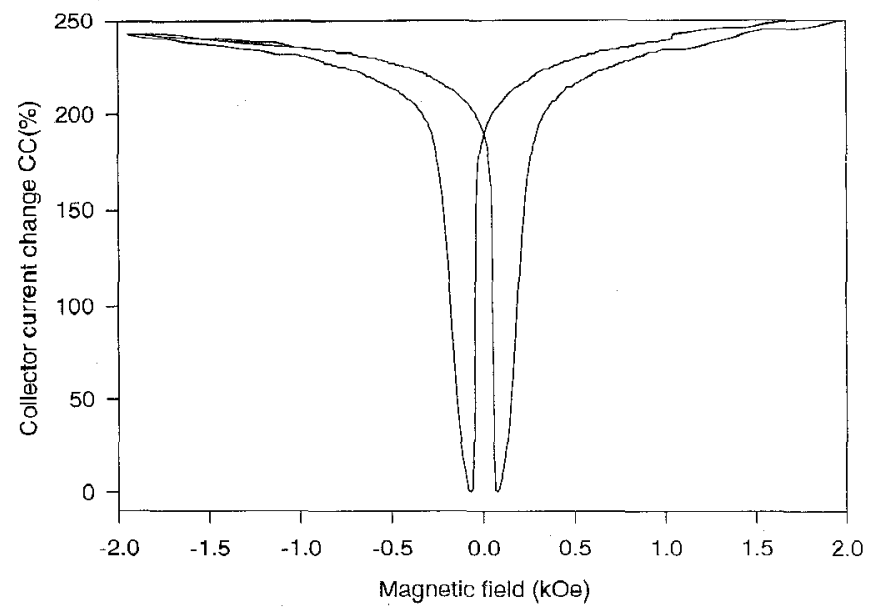

FIG. 3. The collector current change of the $\mathrm{Si}-(\mathrm{Cu} 2 \mathrm{~nm} / \mathrm{Co} 1.5 \mathrm{~nm})_{4}-\mathrm{Si}$ spinvalve transistor at $77 \mathrm{~K}$.

Because of the low barrier height and large area of the collector, the leakage current is quite large $\left(I_{\text {leak }} \approx 30 \mu \mathrm{A}\right)$ and exceeds the hot electron collector current for an injection current of $100 \mathrm{~mA}$. Therefore collector current measurements have been performed at $77 \mathrm{~K}$, reducing the leakage current to very small values. Typical GMR characteristics of a second peak $\mathrm{Co} / \mathrm{Cu}$ multilayer, such as saturation field and hysteresis, are observed. The value at coercive field is $0.1 \mu \mathrm{A}$, at $+/-10$ $\mathrm{kOe}(796 \mathrm{kA} / \mathrm{m}) \quad 0.5 \mu \mathrm{A}$, resulting in more than $390 \%$ collector current change. The corresponding CIP-MR value of the implemented multilayer was only $3 \%$ in $10 \mathrm{kOe}(796$ $\mathrm{kA} / \mathrm{m}, 77 \mathrm{~K})$. The large values of $\mathrm{CC}(\%)$ and $\mathrm{J}_{\mathrm{e}} / \mathrm{J}_{\mathrm{c}}$ indicate a short $\lambda_{\downarrow(\uparrow)}$ (of the order of $0.5(1) \mathrm{nm}$ ).

In [18] a calculation has been made of the enhancement of GMR as a function of the electron energy above the Fermi level in $\mathrm{Co} / \mathrm{Cu}(100)$ multilayers using a calculation within the Kubo formalism in which the electronic bandstructure has been taken into account. There is an asymmetry in the DOS for majority and minority spin, which is typical for ferromagnetic metals. The larger velocity of hot majority electrons travelling increasingly in the sp band above $E_{F}$ results in an increasing GMR with electron energy up to about $1 \mathrm{eV}$. Above $1 \mathrm{eV}$ the asymmetry will diminish and GMR is expected to decrease.

\section{APPLICATION PROSPECTS}

As a consequence of the direct MFP dependence of the transmission across the base, the spin-valve transistor allows quantification of spin dependent electron MFPs $\lambda_{\downarrow}(\uparrow)$ of the individual layers and interfaces. The relation between the change in collector current and the mean free path ratio $\lambda_{\uparrow} / \lambda_{\downarrow}$, expected from the transport equations [10] is illustrated in Fig. 4. The relative change in collector current $\mathrm{CC}(\%)$ and the relative change in perpendicular hot-electron spin valve effect in the base CPP-MR(\%) are plotted versus the MFP ratio $\lambda_{\uparrow} / \lambda_{\downarrow} \cdot \mathrm{CC}(\%)=\left(\mathrm{J}_{\mathrm{P}-\mathrm{J}}\right) / \mathrm{J}_{\mathrm{AP}}$ can either be calculated using $W / \lambda_{\downarrow}[7]$ or using $W / \lambda_{\uparrow}$ : 


$$
C C(\%)=\cosh \left(\frac{W}{2 \lambda_{\uparrow}}\left(1-\frac{\lambda_{\uparrow}}{\lambda_{\downarrow}}\right)\right)-1 .
$$

Since $W / \lambda_{\uparrow}$ represents maximum output, it is the appropriate value for signal to noise calculations. As shown in Fig. 4, the structure may amplify the intrinsic perpendicular hot-electron MR effect strongly, depending on W/ $\lambda$ of the used multilayer.



FIG. 4. Transmission characteristics of the spin-dependent transport. Notice the difference with [10].

The ratio $\mathrm{J}_{\text {leakage }} / \mathrm{J}_{\mathrm{cc}}$ and, for applications, the desired output level of the spin valve transistor, set an upper limit to $W / \lambda_{\uparrow}$. Since the collector current decreases with $W / \lambda_{\uparrow}$ and the relative collector current change increases with $W \lambda_{\uparrow}$, a figure of merit may be calculated by setting the relation between the noise level, the output current level and the output current change to an optimum. In Fig. 4 it is seen that even with a very small attenuation factor $\left(W / \lambda_{\uparrow}=1\right.$ or $\left.e^{-1} \cong 0.4\right)$, the collector current change is still very large, certainly in comparison with the corresponding CIP value. This means that with a suitable base multilayer or sandwich (with large electron mean free path or small thickness) a large collector current can be combined with a large change. From (1) it is also seen that $\mathrm{CC}(\%)$ does not depend on additional nonmagnetic layer thicknesses. The achievable sensitivity is mainly determined by the signal to noise ratio SNR of the device. The main noise source is partition noise and can be represented by a collector shot noise generator $i_{c}=\sqrt{ }\left(2 q I_{c} d f\right)$ from which an indication of the SNR can be calculated. The magnetic field dependent output is not a voltage as in a four point measurement, but a high impedance collector current source. Also, the noise sources of the SVT and the MR resistor are different: in the SVT partition noise prevails, in the resistor thermal noise. A more detailed analysis is currently under study,

\section{PREPARATION OF THE SVT}

In general, it is very difficult to grow device quality semiconductor material on top of a metal. To circumvent this problem we presented direct bonding in air to obtain a single crystal emitter [10]. In direct bonding two flat, smooth and clean materials can form a permanent electrical and mechanical connection by means of spontaneous adhesion [19]. In this way a sawn piece of silicon was bonded in air directly after sputtering the multilayer (See Fig.1). This bond was formed primarily by vanderWaals forces and hydrogen bonds between adsorbed water molecules and was too weak $\left(\approx 5 \mathrm{~kg} / \mathrm{cm}^{2}\right)$ to allow further lithographical processing. For the spin-valve transistor small sizes are required for room temperature operation. Also, the current gain of non-magnetic metal base transistors was still quite low $(5 \%$ at room temperature), partly due to the interface states at the imperfectly bonded emitter Schottky barrier which caused insufficient electron injection properties. We now introduce a new technique, namely vacuum metal deposition bonding.

For the preparation Prime quality $\mathbf{n}(100) \mathrm{Si}$ wafers and $\mathrm{n}(111) \mathrm{Ge}$ wafers are used. The preparation of the silicon is according to the following scheme (class 100 cleanroom condition): Dry oxidation $(30 \mathrm{~nm})$, photoresist protection, sawing of the substrates into $12 \times 21 \mathrm{~mm}$ parts, $\mathrm{HNO}_{3}$ cleaning, DEMI rinse, HF $2 \%$ dip to remove native oxide from sawing particles, $7 \mathrm{~min}$. TMAH (Tetra Methyl Ammonium Hydroxide) etching of $\mathrm{Si}$ particles at $90^{\circ} \mathrm{C}, \mathrm{HF}$ $50 \%$ strip of $30 \mathrm{~nm}$ oxide, DEMI rinse and dry $\mathrm{N}_{2}$ blow dry. The Ge is cleaned as follows: photoresist protection, sawing, $\mathrm{HF}(50 \%): \mathrm{H}_{2} \mathrm{O}_{2}(33 \%)$ 1:10 for Ge fragment etch, $\mathrm{HNO}_{3}$ clean, DEMI rinse, HF dip, DEMI rinse and again blow dry. A Teflon vacuum pipette is used to manipulate the samples without contaminating the surface. Following this procedure the substrates (a) and (b) are attached to the rotatable arms of a bond tool using clips (Fig. 5). The bond tool is made mainly from aluminum and stainless steel. Before loading the system into the sputtering chamber, a spring is loaded. Via magnetic coupling beam (c) is lifted and the spring is released. Substrates (a) and (b) then move towards each other during sputtering of the metal layer. A flywheel and gears which are connected to the rotatable arms dampen the action and in about 0.5 seconds after launch the substrates come into contact. One of the two substrate holders is attached to the arm in a flexible way to permit parallel alignment during contacting. In Fig. 6, as an example, a cross section TEM picture of two silicon substrates bonded with a $30 \mathrm{~nm}$ Ti layer $(2 \times 15 \mathrm{~nm})$ sputtered using a background pressure $\mathrm{p}=5^{*} 10^{-8}$,

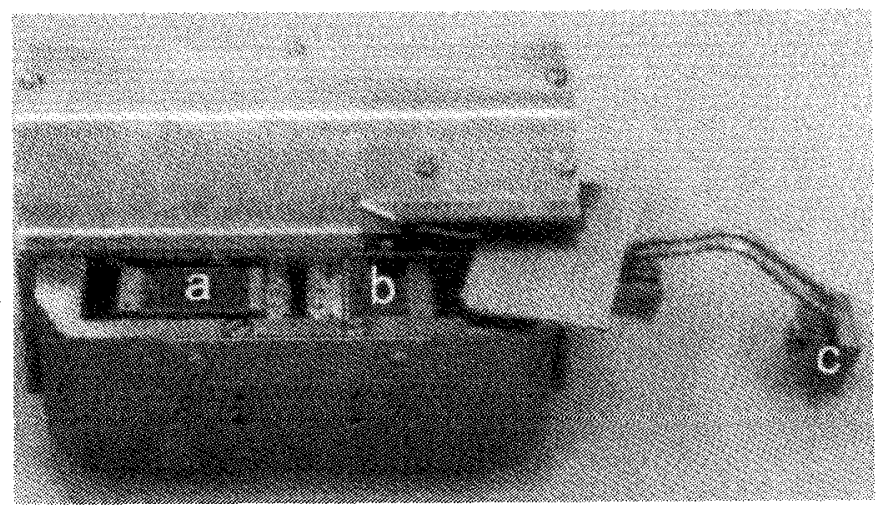

Fig. 5 Spring driven substrate rotator, substrates (a) and (b) and beam (c). 


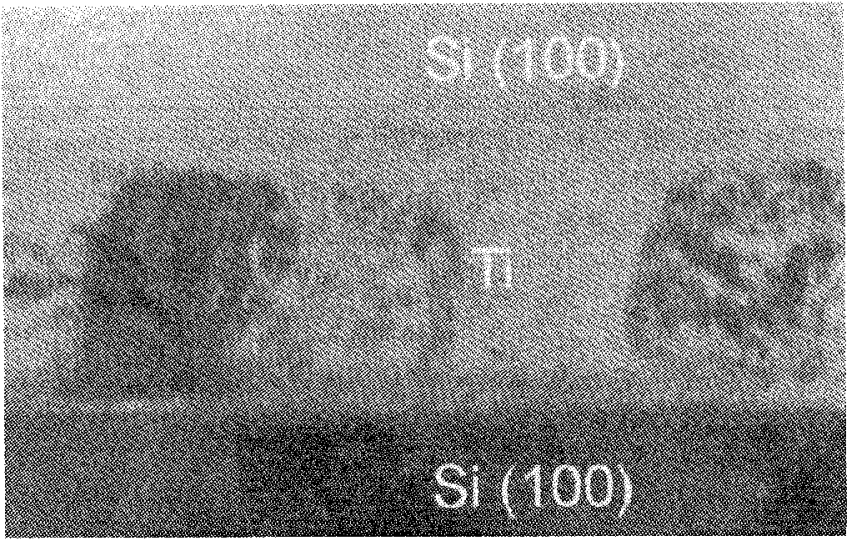

Fig. 6 TEM cross section of Si-Ti30nm-Si.

an argon pressure $\mathrm{p}_{\mathrm{Ar}}=7 * 10^{-3} \mathrm{mbar}$ and Power $\mathrm{P}=30 \mathrm{~W}$. As can be seen, large crystalline regions traversing the entire $\mathrm{Ti}$ film are observed. The (poly)crystallinity is also confirmed by electron diffraction patterns. An interface cannot be distinguished, apparently the $\mathrm{Ti}$ atoms are still very mobile during the bonding time and recrystallisation takes place during bonding. More details about the metallurgical properties of the bond will be reported upon later [20].

The basic law governing the bonding between the substrates is the reduction of the surface free energy. Stress created by roughness compensation during matching of the surfaces counteracts this drive, but for microroughnesses smaller than about $2 \mathrm{~nm}$ complete bonding proceeds spontaneously, i.e. without force at room temperature. The bonding strength was larger than the fracture strength of the silicon. The yield of the bonding procedure is surprisingly good, $100 \%$ under standard conditions. For the development of spin-valve transistors into smaller devices, grinding, polishing, lithography and wet chemical etching has been performed successfully. Vacuum bonded Si-Au-Ge metal base transistor structures with different device areas are shown in Fig 7. Following this, the preparation of small area $(10 \mu \mathrm{m})$ room temperature operating spin-valve transistors is

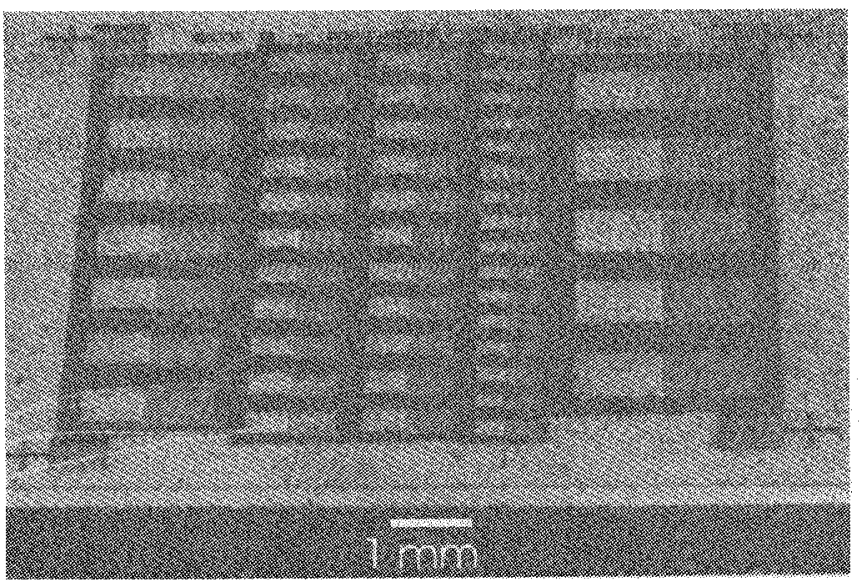

Fig. 7 SEM picture of lithographically processed $\mathrm{Si}-\mathrm{Au}-\mathrm{Ge}$ metal base transistor structures.

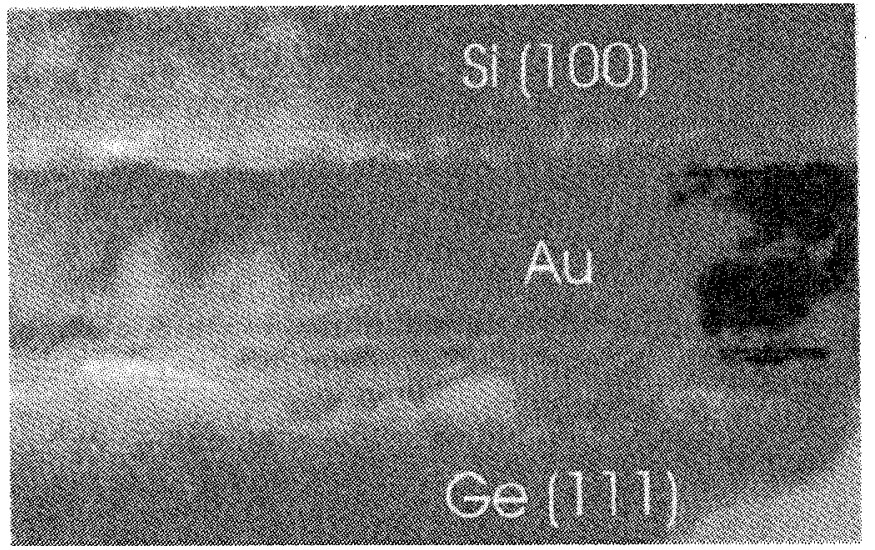

Fig. 8 TEM picture Si-Au $14 n m-G e$ metal base transistor structure showing indiffusion of gold into Ge.

projected, using both $\mathrm{Si}, \mathrm{Ge}$ and GaAs. Metal base transistor structures without magnetic layers are currently under investigation to determine the transistor behaviour apart from the perpendicular spin-valve transport.

A cross section TEM picture of the $\mathrm{Si}-\mathrm{Au}-\mathrm{Ge} \mathrm{MBT}$ is shown in Fig. 8. It reveals again crystals traversing the interface, but also diffused regions of gold into the germanium, presumably aided by the high energy of the sputtered adatoms and diffusive nature of gold. Nevertheless, electrical measurements have shown good emitter and collector Schottky I-V characteristics (although $0.7 \mathrm{eV}$ barrier height instead of $0.8 \mathrm{eV}$ which is expected for Au-Si) and very reproducible hot electron collector currents.

An example of a transistor characteristic of the SiAu14nm-Ge $(350 \times 350 \mu \mathrm{m})$ device is shown in Fig. 9. Ge is used as collector material to obtain barrier height difference between emitter and collector. The current gain is still quite small, as may be expected from the diffused regions at the interfaces (Fig. 8), but the versatility of the bonding technique is expected to allow rapid improvement. Especially lower energy deposition methods of the metal, as in molecular beam epitaxy, or thermal- and e-beam evaporation may show major

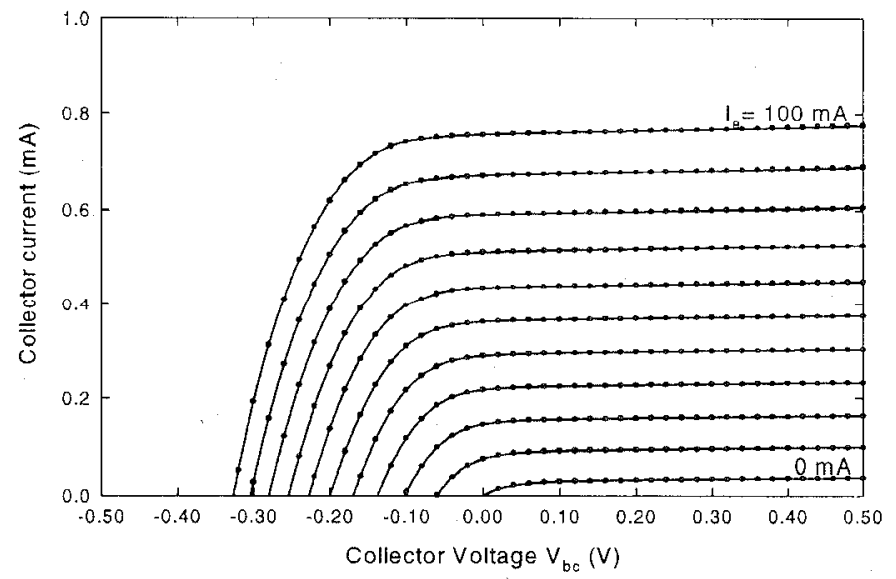

Fig. 9 Common base characteristic of lithographically processed vacuum bonded $\mathrm{Si}$-Au $14 \mathrm{~nm}$-Ge metal base transistor structure. $\mathrm{I}_{\mathrm{c}}$ is plotted versus $V_{b x}$ for $\mathrm{I}_{e}=0-100 \mathrm{~mA}$ in $10 \mathrm{~mA}$ steps. 
improvements of the interface sharpness and current gain

For the spin valve transistor reproducibility is more important. In the realised metal base transisitors the transfer characteristics varied less than $10 \%$ from device to device, which promises good prospects for future investigations. As expected, the hot electron collector current is independent of device area $\left(0.1 \mathrm{~mm}^{2}-1 \mathrm{~mm}^{2}\right.$, see also Fig. 7), whereas the Schottky leak current is proportional to this area. Many injectors and collectors may now be tried for the development of high gain metal base transistors and spin-valve transistors: tunnel injection using a $\mathrm{Si}-\mathrm{SiO}_{2}$ structure, bandgap tailored GaAlAs injectors, high mobility semiconductors, shallow implants and so on. The advantages of semiconductor and semiconductor-oxide injectors as compared to metal-oxidemetal (MOM) injectors are the high maximum current density (up to $10^{5} \mathrm{~A} / \mathrm{cm}^{2}$ ), large breakdown voltage and reproducible IV characteristics.

\section{CONClusions}

In conclusion, the spin-valve transistor obtains important properties as a spectroscopic measurement tool of the CPPGMR: the electron energy dependence of the spin-valve effect can be investigated in an important uncovered electron energy range. The collector current is a direct measure of the perpendicular hot electron mean free paths $\lambda_{\uparrow}(\downarrow)$ and is strongly dependent on a magnetic field. For read head applications soft spin valve sandwiches with long electron mean free paths or small thicknesses should be implemented for large collector current variation in low fields.

Full strength bonding at room temperature has been demonstrated by mating of silicon substrates during sputter deposition of a metallic film. TEM pictures show a buried metal film without an interface. This new bonding technique allows preparation of small devices and with it room temperature operating spin-valve transistors. Other applications of the new bonding technique lie in the field of micromechanics, integration of optoelectronic integrated circuits (OEICs) e.g. III-V and Si wafers, and bonding in general which may take advantage of the extremely strong bond with its chemically resistant, thermally and electrically conducting and, using magnetic materials, magnetic flux preserving link.

\section{REFERENCES}

[1] M.N. Baibich, J.M. Broto, A.Fert, F, Nguyen Van Dau, F. Petroff, P. Etienne, G. Creuzet, A. Friederich, and J. Chazelas, "Giant magnetoresistance of $(001) \mathrm{Fe} /(001) \mathrm{Cr}$ magnetic superlattices" Phys. Rev. Lett. vol. 61, pp. 2472-2475, 1988.

[2] B. Dieny, "Giant magnetoresistance in spin-valve multilayers," J. Magn. Magn. Mater, vol. 136, pp. 335-359, 1994.

[3] W.P. Pratt, Jr., S.F. Lee, J.M. Slaughter, R. Loloee, P.A. Schroeder, and J. Bass, "Perpendicular giant magnetoresistance of $\mathrm{Ag} / \mathrm{Co}$ multilayers," Phys. Rev. Lett. vol. 66, pp. 3060-3066, 1991.

[4] M.A.M. Gijs, S.K.J. Lenczowski, and J.B. Giesbers, "Perpendicular giant magnetoresistance of microstructured $\mathrm{Fe} / \mathrm{Cr}$ magnetic multilayers from 4.2 300K," Phys. Rev. Lett, vol. 70, pp. 3343-3346, 1993.

[5] L. Piraux, J.M. George, J.F. Despres, C. Leroy, E. Ferain, R. Legras, K Ounadjela and A. Fert, "Giant magnetoresistance in magnetic multilayered nanowires," Appl. Phys. Lett, vol. 65, pp. 2484-2486, 1994.

[6] A. Blondel, J.P. Meier, B. Doudin and J.P. Ansermet," Giant magnetoresistance of nanowires of multilayers," Appl. Phys. Lett., vol. 65, pp. 3019-2l, 1994.

[7] Shinjo, T., Ono, T, and Yamamoto, H., Colloqium digest of the 14th international Collogium on magnetic films and surfaces, Düsseldorf: Heinrich Heine Universität, 1994, pp.245-247.

[8] M.A.M. Gijs, M.T. Johnson, A. Reinders, P.E. Huisman, R.J.M. Vandeveerdonk, S.K.J. Lenczowski and R.M.J. Vangansewinkel," Perpendicular giant magnetoresistance of $\mathrm{Co} / \mathrm{Cu}$ deposited under an angle on grooved substrates," Appl.Phys.Lett. vol. 66, pp. 1839-1841, 1995.

[9] M.A.M. Gijs and G.E.W. Bauer, "Perpendicular Giant Magnetoresistance of Magnetic Multilayers," (Review), in press.

[10] D.J.Monsma, J.C.Lodder, Th.J.A. Popma and B.Dieny, "Perpendicular hot electron spin-valve effect in a new magnetic field sensor: The spin valve Transistor," Phys. Rev. Letters, vol. 74, pp. 5260-5263, 1995.

[11] R. Feder Polarized Electrons in Surface Physics, Advanced Series in Surface Science, vol. 1, World Scientific, Singapore, 1985

[12] R.J. Celotta, J. Unguris, and D.T. Pierce, "Hot electron spin-valve effect in coupled magnetic layers," J. Appl. Phys. vol. 75, pp. 6452-6455, 1994.

[13] H.J.Drouhin, A.J. van der Sluijs, Y.Lassaily, and G. Lampel, "Spin dependent transmission of free electrons through ultrathin cobalt layers," $J$. Appl. Phys. vol. 79, pp. 4734-4739, 1996.

[14] J.C.Gröbli, D.Oberli, and F. Meier, "Crucial tests of spin filtering," Phys. Rev. B, vol. 52, pp. R13095-R13097, 1995 ,

[15] J.S. Moodera and L. R. Kinder, "Ferromagnet-insulator-ferromagnet tunneling: spin-dependent tunneling and large magnetoresistance in trilayer junctions," J. Appl. Phys., vol.79, pp. 4724-4729, 1996,

[16] H.C. Siegmann, "Surface and 2D magnetism with spin-polarized cascade electrons," Surface Science, vol. 307-309, pp. 1076-1086, 1994,

[17] Tillmann D., "Very low energy spleed from Fe(001)," Z Phys.BCondensed matter, vol. 77, pp. 1-5, 1989,

[18] E.Y.Tsymbal and D.G.Pettifor, "The influence of spin-independent disorder on giant magnetoresistance," $J$. Phys.: Condens. Matter, vol. 8, pp. L569-L575, 1996.

[19] U. Gösele, H. Stenzel, M. Reiche, T. Martini, H. Steinkirchner and Q.Y. Tong, "History and future of semiconductor wafer bonding," Solid Sate Phenomena vol. 47-48, pp. 33-44, 1996 and references therein.

[20] T. Shimatsu, D.J. Monsma, R. Mollema and J.C. Lodder, unpublished. 